\title{
Validez diagnóstica de la biopsia intraoperatoria en cirugía de lesiones mamarias palpables
}

\author{
Enrique Bellolio J ${ }^{\text {lab }}$, Pablo G uzmán $\mathbf{G}^{\text {1b }}$, Juan 0 rellana $C^{2 c}$, \\ Juan Carlos Roa $\mathrm{S}^{1}$, Miguel Villaseca $\mathrm{H}^{1}$, Juan Carlos Araya $\mathbf{0}^{1}$, \\ O scar Tapia Ela, Viviana Pineda $N^{3}$.
}

\section{Diagnostic value of frozen section biopsy during surgery for breast lesions or neoplasms}

Background: During the surgical treatment of breast neoplasms (benign or malignant), frozen section biopsy is frequently requested to assess the kind of lesion and determine the surgical margins. Aim: To assess the diagnostic yield of frozen section breast biopsy. Material and methods: All the pathological reports of frozen section biopsies and definitive biopsies of 337 women aged 26 to 88 years, operated for suspected breast neoplasms between 2002 and 2006, were reviewed. The sensitivity, specificity and predictive value of frozen section biopsy, were calculated using the definitive biopsy as the gold standard. Results: The definitive biopsy confirmed the presence of cancer in 290 women (86\%). There were two false negative $(0.59 \%)$ and no false positive frozen section biopsies for cancer detection. The sensibility for cancer detection was $99.3 \%$ and the specificity $100 \%$. The positive predictive value was $100 \%$ and the negative predictive value $96.1 \%$. The diagnosis of phyllodes tumor was missed by frozen section biopsy in three cases. The margins were informed in the 258 frozen section biopsies (79\%) and in 59 cases (18\%), these were positive for cancer. Conclusions: Frozen section biopsy is useful and reliable for cancer detection and margin status assessment in breast cancer surgery (Rev Méd Chile 2009; 137: 1173-8).

(Key words: Biopsy; Breast neoplasms; Frozen sections)

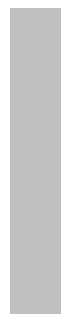

Recibido el 24 de diciembre, 2008. Aceptado el 3 de agosto, 2009.

Trabajo financiado por la Dirección de Investigación de la Universidad de La Frontera (DIUFRO).

${ }^{1}$ Departamento de Anatomía Patológica, Universidad de La Frontera. ${ }^{2}$ Centro de Excelencia, Capacitación, Gestión e Investigación para la Salud Basada en Evidencias (CIGES), Facultad de Medicina, Universidad de La Frontera. ${ }^{3}$ Departamento de Cirugía y Traumatología, Universidad de La Frontera. Temuco, Chile.

${ }^{\text {a }}$ Residente

b Magíster en Epidemiología Clínica

${ }^{\mathrm{c}}$ Bioestadístico

$\overline{\text { Correspondencia a: Dr. Enrique Bellolio J. Departamento }}$ de Anatomía Patológica, Facultad de Medicina, Universidad de La Frontera. Manuel Montt 112. Código Postal 478-1176. Temuco, Chile. E mail: ebellolio@gmail.com 
$\mathrm{E}^{\prime}$ cáncer de mama es la primera causa de muerte por cáncer en la mujer a nivel mundial, con una tasa de mortalidad estandarizada por edad de 13,2 por cien mil mujeres y su incidencia estandarizada por edad de 37,4 por cien mil mujeres $^{1}$. Al año fallecen 1,2 millones de mujeres por esta causa, representando $14 \%$ del total de muertes por cáncer a nivel mundial. La incidencia de cáncer de mama ha aumentado en todo el mundo, principalmente en los países en vías de desarrollo ${ }^{2}$. En Estados Unidos de Norteamérica la tasa de incidencia anual es de 127,8 por cada cien mil mujeres y la tasa de mortalidad es de 25 por cada cien mil mujeres ${ }^{3}$. En Chile la tasa de mortalidad por cáncer de mama en el año 2005 fue de 14,2 por cada cien mil mujeres, tasa que en la región de La Araucanía fue de 11,1 por cada cien mil mujeres, con una tendencia al alza nacional en los últimos 15 años ${ }^{4}$. Al analizar las causas de muerte específicas en mujeres según grupos etarios, tenemos que el cáncer de mama ocupa el cuarto lugar entre los 20 a 44 años y el tercer lugar entre los 45 a 64 años. En ambos grupos, es la segunda causa de muerte por cáncer, siendo sobrepasado en el primer grupo etario por el cáncer de cuello uterino y en el segundo grupo etario por el cáncer de vesícula biliar ${ }^{3}$.

La biopsia diferida (BD) es el patrón de oro para el diagnóstico de esta patología, procedimiento que consiste en la obtención de tejido mamario para su posterior análisis bajo microscopio de luz por un anatomopatólogo. La biopsia intraoperatoria (BIO) es el examen que se realiza durante la cirugía, en un corte de congelación de tejido fresco con tinción abreviada de hematoxilina-eosina. Las utilidades de la BIO se resumen en: a) Permitir diagnóstico intraoperatorio de la presencia, naturaleza (neoplásica o no-neoplásica) y carácter (benigno, maligno o incierto) de una lesión determinada; b) Determinar la presencia de lesión en los bordes quirúrgicos; y c) Asegurar la obtención de material adecuado para su posterior estudio. Un tiempo promedio considerado adecuado es de 20 min y el nivel de concordancia entre la $\mathrm{BIO}$ y la BD debiera ser superior a $97 \% 5$. En el último tiempo la utilización de la biopsia por punción (BPP) ha desplazado a la citología por aspiración, dado que la primera permite determinar si una lesión es in situ o infiltrante, evaluar la presencia de microcalcificaciones y el diagnóstico de lesiones benignas ${ }^{6}$. La BPP es un examen que se ha incrementado en las pacientes que serán tratadas con cirugía, disminuyendo el número de BIO necesarias para determinar el carácter de la lesión, relegando la utilidad de la BIO para evaluar los márgenes quirúrgicos de tumorectomías y en la búsqueda de metástasis del linfonodo centinela 7 .

En Chile, según nuestra revisión con buscador www.scielo.cl y www.pubmed.com, no hay publicaciones que hagan mención a la validez diagnóstica de la BIO en cirugía de lesiones mamarias, sólo hay una publicación de correlación entre ambas biopsias pero en cáncer de ovario ${ }^{8}$ y otra en cáncer de tiroides 9 . Dado lo anterior, el objetivo del presente trabajo es poder evaluar la validez diagnóstica de la BIO para la detección de cáncer y evaluación de márgenes quirúrgicos en biopsias de lesiones mamarias, con la finalidad de conocer la realidad local, estandarizar el procedimiento e implementar mejoras en caso de ser necesario.

\section{MATERIAL Y MÉTODO}

Se diseñó un estudio de prueba diagnóstica, transversal, retrospectivo, con análisis estadístico descriptivo y analítico ${ }^{10}$, con muestreo no probabilístico consecutivo de todas las BIO realizadas por lesiones mamarias de mujeres intervenidas quirúrgicamente en la Unidad de Anatomía Patológica (UAP) del Hospital Dr. Hernán Henríquez Aravena de Temuco, en el período comprendido entre el 1 de enero de 2002 y el 31 de diciembre de 2006. Las cirugías que se incluyeron son aquellas realizadas en lesiones mamarias palpables. Los tipos de piezas quirúrgicas incluidas en el estudio fueron la mastectomía radical y tumorectomía, esta última con margen de seguridad macroscópico por palpación e identificación de márgenes quirúrgicos. Los márgenes quirúrgicos identificados son: anterior, medial y posterior.

Se revisaron todos los informes diagnósticos histopatológicos de $\mathrm{BIO}$ y $\mathrm{BD}$, con los que se confeccionó una base de datos con las siguientes variables (tipo, unidad de medida): número de biopsia (nominal), edad paciente (continua, en años), diámetro mayor de muestra recibida (continua, en mm), diámetro mayor del tumor (continua en $\mathrm{mm}$ ), distancia entre la lesión y margen de resección (continua en $\mathrm{mm}$ ), diagnóstico BIO (nominal), diagnóstico de cáncer en BIO (dicotómi- 
ca), diagnóstico de BD (nominal), diagnóstico de cáncer en BD (dicotómica), patólogo examinador (ordinal), tiempo de realización de BIO (continua, en min). Los exámenes fueron realizados en forma individual por los integrantes del equipo de patólogos de la UAP, conformado por 5 profesionales. Se tabuló los datos en tablas de 2 × 2 y fueron analizados con el programa estadístico Stata 9.0.

Método de realización de BIO. Una vez recibida la muestra en la UAP, se asigna un número de biopsia y se traslada a la sala de dictado macroscópico, donde es evaluada por el anatomopatólogo y residente de turno. En el examen macroscópico, se mide, secciona y toma muestra para evaluar la superficie y presencia de lesiones sospechosas. Se realiza citología y selección del fragmento de tejido a examinar. La muestra es montada en un tope de aluminio con gel de montaje (Tissue Tek $^{\circledR}$ ), para luego ser congelada a $-24^{\circ} \mathrm{C}$ y cortada en el crióstato (Microm ${ }^{\circledR}$ modelo HM 505 E). Los cortes son de 8 a 12 micrones de espesor, obteniendo de 1 a 2 cortes que son teñidos manualmente con hematoxilina-eosina en protocolo abreviado que tarda $3 \mathrm{~min}$, para luego ser examinados en microscopio de luz. El informe histopatológico es enviado vía fax a pabellón central. La BD se obtiene de muestras de tejido fijado previamente por al menos $24 \mathrm{~h}$ con formalina al $4 \%$ tamponada. Posteriormente, las muestras mediante procesamiento histológico automatizado y tinción con hematoxilina-eosina, son finalmente entregadas al patólogo en un proceso que tarda, en promedio, 24 h más.
Los informes obtenidos fueron clasificados según la presencia de cáncer en $\mathrm{BD}$ en malignos y benignos, para luego ser comparados con los informes de las BIO para cada caso en particular. La distancia se midió en mm desde el punto de menor distancia entre el borde de la lesión y el margen de resección lateral o profundo.

\section{Resultados}

Se realizaron 337 biopsias intraoperatorias en cirugías de mama en el quinquenio revisado. Todas corresponden a biopsias de mujeres, con un promedio de edad de 56 años (valor mínimo 26 años y valor máximo de 88 años). En la Tabla 1 se muestran los diagnósticos de $\mathrm{BD}$ con sus respectivas frecuencias absolutas y relativas. De las 337 biopsias realizadas, en 290 se informó cáncer en la BD, lo que representa 86\%.

En la Tabla 2 se describen las variables cuantitativas según diagnóstico de cáncer en la biopsia diferida, destacando que la edad promedio fue 12 años mayor en el grupo con cáncer ( $p$ $<0,01$ ) y el tamaño del tumor en promedio $6,6 \mathrm{~mm}$ mayor en el grupo con cáncer ( $\mathrm{p}<0,01)$. El tiempo promedio que toma la biopsia rápida en la UAP en cirugías de lesiones mamarias fue de $10 \mathrm{~min}$ IC 95\% [10,06-11,36]; no se encontraron diferencias estadísticamente significativas en tiempo promedio que toma el diagnóstico si la BIO es positiva o negativa para cáncer ( $\mathrm{p}=0,08)$, así como tampoco en el tamaño de la muestra recibida $(\mathrm{p}=0,17)$.

Tabla 1 Distribución según diagnósticos

\begin{tabular}{|lrllr|}
\hline \multicolumn{2}{|c}{ Lesiones benignas } & & & \multicolumn{2}{c|}{ Lesiones malignas } & n \\
Diagnóstico & $\mathbf{n}$ & $(\%)$ & Diagnóstico & $273(94)$ \\
\hline Fibroadenoma & 28 & $(60)$ & Carcinoma de células de los conductos & $5(1,7)$ \\
Tumor phyllodes & 5 & $(11)$ & Tumor phyllodes* & $4(1,4)$ \\
Mastopatía fibroquística & 4 & $(9)$ & Carcinoma mucinoso & $3(1)$ \\
Papiloma intraductal & 3 & $(6)$ & Carcinoma lobulillar & $2(0,7)$ \\
Fibrosis mamaria & 2 & $(4)$ & Carcinoma in situ & $1(0,3)$ \\
Adenosis periductal & 1 & $(2)$ & Carcinoma medular & $1(0,3)$ \\
Cicatriz radiada & 1 & $(2)$ & Carcinoma papilar & 1 \\
Comedomastitis & 1 & $(2)$ & Sarcoma pleomórfico no especificado & \\
Fibromatosis & 1 & $(2)$ & & \\
Hiperplasia ductal con atipías & 1 & $(2)$ & & $290(100)$ \\
Total & 47 & $(100)$ & & \\
\hline
\end{tabular}

$\mathrm{n}$ =número de casos. *Borderline y malignos. 
Tabla 2 Comparación de variables continuas según resultado de BD

\begin{tabular}{|lccc|}
\hline & & Biopsia diferida \\
& $(+)$ cáncer & 46 & $<$ Valor $\mathbf{p}$ \\
\hline Edad (años) & 58 & 10,55 & $<0,01$ \\
Tiempo (min) & 11,85 & 59,5 & 0,08 \\
Tamaño tejido recibido (mm) & 55,6 & 22,2 & 0,17 \\
Tamaño tumor (mm) & 28,8 & $<0,01$ \\
\hline
\end{tabular}

En las Tablas 3 y 4 se muestra la prueba de validación diagnóstica realizada, donde la sensibilidad alcanzada para la detección de cáncer de mama de la BIO fue de 99,3\%; la especificidad de 100\%; según la prevalencia de cáncer observada de 86\%, el valor predictivo positivo de $100 \%$ y el valor predictivo negativo de 96,1\%. La exactitud de la BIO fue de 99\%. El porcentaje de concordancia alcanzado fue de 98,81\% con índice kappa de 0,952, este último indica una concordancia casi perfecta ${ }^{11}$. Al analizar las tasas de probabilidad, que en conjunto son el mejor indicador de una prueba diagnóstica, se tiene que el valor de la tasa de probabilidad positiva no se puede calcular dado que la especificidad de la $\mathrm{BIO}$ es de $100 \%$ (cero falso positivo) y la tasa de probabilidad negativa es 0,01 . Sólo hubo 4 falsos negativos $(1,18 \%)$ para la BIO respecto de lo informado en la biopsia diferida, dos lesiones benignas y dos lesiones malignas. Respecto de la sensibilidad para detección de cáncer en la BIO, sólo hubo dos falsos negativos. Cabe mencionar que en un caso se informó fibroadenoma en la $\mathrm{BIO}$ y en la BD fue diagnosticado como tumor phyllodes maligno y el otro caso fue informado como cicatriz radiada en la BIO y el diagnóstico de la

Tabla 3. Correlación de BIO y diferida

\begin{tabular}{|lccr|}
\hline BIO & $(+)$ cáncer & $\begin{array}{c}\text { Biopsia diferida } \\
(-) \text { cáncer }\end{array}$ & Total \\
\hline (+) cáncer & 286 & 0 & 286 \\
(-) cáncer & 2 & 49 & 51 \\
Total & 288 & 49 & 337 \\
\hline
\end{tabular}

Tabla 4. Prueba de validación de BIO en detección de cáncer

\begin{tabular}{|lcc|}
\hline & Valor & IC 95\% \\
\hline Sensibilidad & $99,3 \%$ & $97,5-99,9 \%$ \\
Especificidad & $100 \%$ & $92,7-100 \%$ \\
Área COR (Sen+Esp) & 1 & $0,99-1$ \\
Valor predictivo (+) & $100 \%$ & $98,7-100 \%$ \\
Valor predictivo (-) & $96,1 \%$ & $86,5-99,5 \%$ \\
Tasa probabilidad (+) & - & - \\
Tasa probabilidad (-) & 0,01 & $0,00-0,03$ \\
Exactitud & $99 \%$ & $98-99,5 \%$ \\
Concordancia & $98,81 \%$ & N/A \\
Índice kappa & 0,952 & N/A \\
\hline
\end{tabular}

IC =Intervalo de confianza; N/A =No aplica. 
BD fue carcinoma in situ. En 258 casos se informó la distancia entre la lesión y los bordes quirúrgicos con un promedio de $3,96 \mathrm{~mm}$ y un rango de 0 a $40 \mathrm{~mm}$; en los restantes 79 casos sólo se informó que no había compromiso de margen de resección. En 59 casos (18\%) se informó la presencia de cáncer en la BIO con compromiso del margen de resección. De éstos en 47 casos se realizó una ampliación de la cirugía en el mismo tiempo quirúrgico, sin solicitud de BIO, siendo dicha ampliación negativa en la BD para todos los casos. La ampliación de cirugía consistió en 35 mastectomías radicales y en 12 ampliaciones del margen de resección, lo que disminuyó las reintervenciones posteriores en estos casos. No hubo casos con margen positivo en $\mathrm{BD}$ y que fueran negativos en BIO. No se encontró diferencia significativa en la correlación diagnóstica según el examinador, dado que sólo fueron 4 falsos negativos.

\section{DisCUSIÓN}

El análisis de los datos nos permite asegurar que la BIO es un examen con validez comprobada para la detección de cáncer de mama en la Unidad de Anatomía Patológica del Hospital Regional, dada su alta sensibilidad y excelente especificidad. El trabajo de Yun et $\mathrm{al}^{12}$, con 13.340 casos, comunicó 0,9\% de falsos negativos en la detección de cáncer por la BIO, similar a lo alcanzado en nuestro estudio. La exactitud de la BIO reportada por Yun fue de 99,1\%, también similar a la alcanzada en nuestro estudio, lo que refuerza que los indicadores de la $\mathrm{BIO}$ en nuestra institución son similares a otros centros de acuerdo a lo descrito en la literatura internacional para la detección de cáncer de mama. Respecto de los casos identificados como falsos negativos en nuestra serie el problema fue con la identificación de tumor phyllodes y con cicatriz radiada, situación que difiere un poco de lo reportado por Yun, donde las mayores dificultades pueden deberse a tratamiento previo con quimioterapia, inflamación crónica granulomatosa, carcinoma papilar bien diferenciado y cicatriz radiada. En nuestro caso la dificultad fue con lesiones benignas (fibroadenomas) que fueron catalogadas posteriormente en la BD como tumor phyllodes malignos o limítrofes.

Los valores de especificidad y tasa de probabilidad negativa de la $\mathrm{BIO}$ son excelentes, lo que permite plantear que la $\mathrm{BIO}$ es una prueba diagnóstica casi tan buena como el patrón de oro representado por la BD.
En nuestra serie se diagnosticaron 10 tumores phyllodes $(2,9 \%)$, de los cuales 5 (1,4\%) fueron tumores catalogados como limítrofes y malignos, valores similares a lo descrito en la literatura ${ }^{13}$. En nuestra serie, tuvimos dificultad para realizar el diagnóstico en la BIO de tumor phyllodes, lo que se debe a las características histológicas que se utilizan para su diagnóstico y clasificación, las que son difíciles de evaluar en la $\mathrm{BIO}^{14}$. El mayor problema clínico que se presenta con los tumores phyllodes tiene relación con su recidiva local; se ha descrito que la recidiva está relacionada con el grado histológico y es de 10\% para los benignos, 20\% para los limítrofes y $23 \%$ para los malignos ${ }^{15,16}$.

También hay asociación entre la recurrencia y el compromiso del borde quirúrgico. En estos tumores la mayor utilidad de la BIO es la evaluación de los márgenes quirúrgicos, con el objetivo de asegurar la resección total de la lesión. En el caso que se diagnosticó cicatriz radiada en la $\mathrm{BIO}$, el estudio seriado de la pieza quirúrgica, permitió detectar la presencia de carcinoma in situ. Patterson y $\mathrm{cols}^{17}$ en lesiones catalogadas como cicatriz radiada observaron carcinoma in situ en 16,9\% en los casos sintomáticos y en $4,7 \%$ de los casos detectados por tamizaje mamográfico. Producto del mayor riesgo de asociación de cicatriz radiada con cáncer, algunos autores recomiendan la extirpación completa de las lesiones ${ }^{18}$.

Con el advenimiento de la BPP, cada vez más frecuente, el diagnóstico de cáncer de mama se conoce previo a la cirugía, sumado a lo anterior la disponibilidad de mamografías de tamizaje permitirá que las lesiones sean diagnosticadas en estadios no tan avanzados, lo que debería disminuir el tamaño de las lesiones, incluso llegando la resección de lesiones no palpables. La sensibilidad y especificidad de la BIO en lesiones no palpables varía según lo señalado en la literatura con una sensibilidad entre $80 \%$ y $91,7 \%$ y una especificidad entre $69 \%$ y $99 \% 19-$ 21. Con lesiones de menor tamaño se podrá disminuir el número de mastectomías totales en favor de tumorectomías con disección axilar dirigida con la $\mathrm{BIO}$ del ganglio centinela ${ }^{22}$. Esto último ha generado una disminución del número de BIO para evaluar el carácter de la lesión, situación ya descrita hace un par de años en centros norteamericanos, donde la biopsia por punción lleva más tiempo ${ }^{23}$. La utilidad de la BIO para evaluar los márgenes de resección ha sido reportada en varios artículos, destacando Tartter et $\mathrm{al}^{24}$, Cendán et $\mathrm{al}^{25}$ y Camp et $\mathrm{al}^{26}$. Cabe destacar 
el trabajo de Cendán et al, quien reportó que la BIO permitió evitar la reintervención quirúrgica en 44,2\% de las pacientes tratadas con cirugía por cáncer de mama.

Para concluir podemos señalar que la $\mathrm{BIO}$ es un método válido y confiable en el diagnóstico intraoperatorio de lesiones mamarias palpables en

\section{REFERENCIAS}

1. Globocan 2002 Software ${ }^{\circledR}$ database September 2005. Available at http://www-dep.iarc.fr/

2. Tavassoéli FA. Pathology and Genetics of Tumors of The Breast and Female Genital Organs. En: Tavassoéli FA y Devilee P editores. Lyon, Francia: International Agency for Research Cancer IARCPress 2003; 9-110.

3. Ries Lag, Melbert D, Krapcho M, Mariotto A, Miller BA, Feuer EJ et al. SEER Cancer StatisticsReview, 1975-2004, National Cancer Institute. Bethesda. Available at http:// seer.cancer.gov/csr/1975_2004/.

4. Departamento de Estadística e Información de Salud, Ministerio de Salud de Chile. Información año 2005. Disponible en: http://www.minsal.cl

5. Rosai J. Surgical Pathology, Ninth Edition. Editorial: USA Mosby 2004; 9-12.

6. Litherland JC. Should Fine Needle Aspiration Cytology in Breast Assessment Be Abandoned? Clinical Radiology 2002; 57: 81-4.

7. Noriaki Wada, Shigeru Imoto, Takahiro Hasebe, Atsushi Ochial, Satoshi Ebihara, Noriyuki Moriyama. Evaluation of Intraoperative Frozen Section Diagnosis of SentinelLymph Nodes in Breast Cancer. Jpn J Clin Oncol 2004; 34: 113-17.

8. Cuello M, Galleguillos G, Zárate C et al. Biopsia rápida por congelación en el diagnóstico de tumores de ovario: correlación diagnóstica según diámetro y peso en tumores de origen epitelial. Rev Méd Chile 1999; 127: 1199-205.

9. Astroza G, González M, Paladines P et al. Correlación entre biopsia rápida operatoria y biopsia diferida de tiroides: Revisión de 10 años en el Hospital Barros LucoTrudeau. Rev Chil Cir 2006; 58: 410-13.

10. Hulley S. Designing Clinical Research, Second Edition. USA: Lippincott Williams \& Wilkins; 2001; 175-91.

11. Sackett D, Haynes B, Guyatt G, Tugwell P. Epidemiología Clínica, Segunda Edición; Editorial Médica Panamericana; 1994; 19-62.

12. Niu Yun, Fu Xi-Lin, Yu Yong, Peizhong Peter Wang, Cao $\mathrm{Xu}-\mathrm{CHEN}$. Intraoperative frozen section diagnosis of breast lesions: a retrospective analysis of 13243 Chinese patients. Chin Med J 2007; 120: 630-5.

13. Parker SJ, Harris SA. Phyllodes tumours. Postgrad Med J 2001; 77: 428-35.

14. Foxcroft LM, Evans EB, Porter AJ. Difficulties in the pre-operative diagnosis of phyllodes tumours of the nuestro centro y que tiene un alto índice de correlación con la BD. Esperamos que a futuro siga siendo parte de la planificación del diagnóstico y tratamiento de las pacientes con lesiones mamarias, especialmente en las lesiones neoplásicas con el objetivo centrado en asegurar los márgenes de resección libres de neoplasia.

breast: A study of 84 cases. The Breast 2007; 16: 27-37.

15. Puay-Hoon Tan, Thiyagarajan Jayabaskar, Khoon-Leong Chuah et al. Phyllodes Tumors of the Breast, The Role of Pathologic Parameters. Am J Clin Pathol 2005; 123: 529-40.

16. Roa JC, Tapia O, Carrasco P, Contreras E, araya JC, Muñoz S, ROA I. Prognostic factors of phyllodes tumor of the breast. Pathol Int 2006; 56: 309-14.

17. Pattersona JA, Scottb M, Andersonb N, Kirka SJ. Radial scar, complex sclerosing lesion and risk of breast cancer. Analysis of 175 cases in Northern Ireland. European Journal of Surgical Oncology 2004; 30: 1065-8.

18. Fasih T, Jain M, Shrimankar J, Staunton M, Hubbard J, GrIFFITH. All radial scars/complex sclerosing lesions seen on breast screening mammograms should be excised. European Journal of Surgical Oncology 2005; 31: 1125-8.

19. Bianchi S, Palli D, Ciatto S, Galui M, Giorgi D, Vezzosi V ET AL. Accuracy and reliability of frozen section diagnosis in a series of 672 nonpalpable breast lesions. Am J Clin Pathol 1995; 104: 358-60.

20. Dorel-Letheo M, Dales JP, García S, Ramuz O, AndracMeYer L, Bonnier P ET AL. Accuracy of intraoperative frozen section diagnosis in non palpable breast lesions: a series of 791 cases. Bull Cancer 2003; 90: 357-62.

21. Weber WP, Engelberger S, Viehl CT, Zanetti-Dallenbach R, Kuster S, Dirnhofer S et al. Accuracy of frozen section analysis versus specimen radiography during breastconserving surgery for nonpalpable lesions. World J Surg 2008; 32: 2599-606.

22. Wada N, Imoto S, Hasebe T, Ochial A, Ebihara S, MoriYama N. Evaluation of Intraoperative Frozen Section Diagnosis of Sentinel Lymph Nodes in Breast Cancer. Jpn J Clin Oncol 2004; 34: 113-7.

23. Laucirica R. Intraoperative Assessment of the Breast. Arch Pathol Lab Med 2005; 129: 1565-74.

24. Tartter P, Kaplan J, Bleiweiss I, Gajdos C, Kong A, Ahmed S ET AL. Lumpectomy margins, reexcision, and local recurrence of breast cancer. Am J Surg 2000; 179: 81-5.

25. Cendán C, Coco D, Copeland E. Accuracy of Intraoperative Frozen-Section Analysis of Breast Cancer Lumpectomy-Bed Margins. J Am Coll Surg 2005; 201: 194-8.

26. Camp E, Mcauliffe P, Gilroy J, Morris C, Lind D, Mendenhall N ET al. Minimizing local recurrence after breast conserving therapy using intraoperative shaved margins to determine pathologic tumor clearance. J Am Coll Surg 2005; 201: 855-61. 